\title{
Cardiovascular beriberi: rare cause of reversible pulmonary hypertension
}

\author{
Joon Hyuk Song, Sang Soo Cheon, Myung Hwan Bae, Jang Hoon Lee, Dong Heon Yang, \\ Hun Sik Park, Yongkeun Cho, Shung Chull Chae \\ Department of Internal Medicine, Kyungpook National University Hospital, Daegu, Korea
}

\begin{abstract}
Cardiovascular beriberi is caused by thiamine deficiency and usually presents as high cardiac output failure associated with predominantly right-sided heart failure and rapid recovery after treatment with thiamine. Because of its rarity in developed countries, the diagnosis can often be delayed and missed. We recently experienced a case of cardiovascular beriberi with pulmonary hypertension which successfully treated with thiamine infusion. A 50-year-old man with chronic heavy alcoholics was refered to our department for dyspnea with mental change. Echocardiography showed marked right ventricular (RV) dilatation and flattening of the interventricular septum with a D-shaped deformation of the left ventricle. Moderate tricuspid valve regurgitation was found and estimated RV systolic pressure was $52 \mathrm{~mm} \mathrm{Hg}$. Because of his confused mentality and history of chronic alcohol intake, neurological disorder due to thiamine deficiency was suspected and intravenous thiamine was administered and he continuously received a daily dose of $100 \mathrm{mg}$ of thiamine. Follow up echocardiography showed marked reduction of RV dilatation and improvement of a D-shaped deformation of the left ventricle. He finally diagnosed as cardiovascular beriberi on the basis of dramatic response to intravenous thiamine. Thiamine deficiency can cause reversible pulmonary hypertension, and can still be encountered in the clinical setting. Thus high index of suspicion is critically needed for diagnosis.
\end{abstract}

Keywords: Beriberi; Thiamine deficiency; Pulmonary hypertension; Heart failure

\section{INTRODUCTION}

Clinical syndromes of thiamine deficiency are classically described as "dry beriberi", in which peripheral neuropathy is the cardinal feature, "wet beriberi", in which cardiac failure predominates, and Wernicke's encephalopathy, which comprises a triad of altered consciousness, ocular signs, and ataxia [1]. Deficiency of this vitamin may have a nutritional cause or occur secondary to alcohol intoxication [2].

Thiamine is considered a clinically important factor with respect to heart function, and its deficiency has been reported to cause heart failure [3-5]. Cardiovascular beriberi usually presents as high cardiac output failure associated with predo-

Received: August 12, 2013; Revised: August 29, 2013; Accepted: September 9, 2013

Corresponding Author: Dong Heon Yang, Department of Internal Medicine, Kyungpook National University Hospital, 130 Dongdeok-ro, Jung-gu, Daegu 700-721, Korea Tel: +82-53-420-6587, Fax: +82-53-426-2046

E-mail: ddhyang@knu.ac.kr minantly right-sided heart failure and rapid recovery after treatment with thiamine [5]. However not all cases display the classical signs and there are no specific laboratory tests that can diagnose the syndrome. Furthermore, because of its rarity in developed countries like Korea, the diagnosis can often be delayed and missed. Therefore, high index of suspicion is needed. We hereby present a case of cardiovascular beriberi with reversible right-sided heart failure and pulmonary hypertension successfully treated with thiamine.

\section{CASE}

A 50-year-old man, a chronic heavy alcoholic, was referred to our department for dyspnea, gait disturbance, and confused mentality. He had been consuming 2 liters of makgeolli (Korean raw rice wine) per day for a year. He was in an oliguric state. On physical examination, he presented a blood pressure of $148 / 82 \mathrm{~mm} \mathrm{Hg}$, a respiratory rate of 26 breaths per minute, and a heart rate of 86 beats per minute. Both 


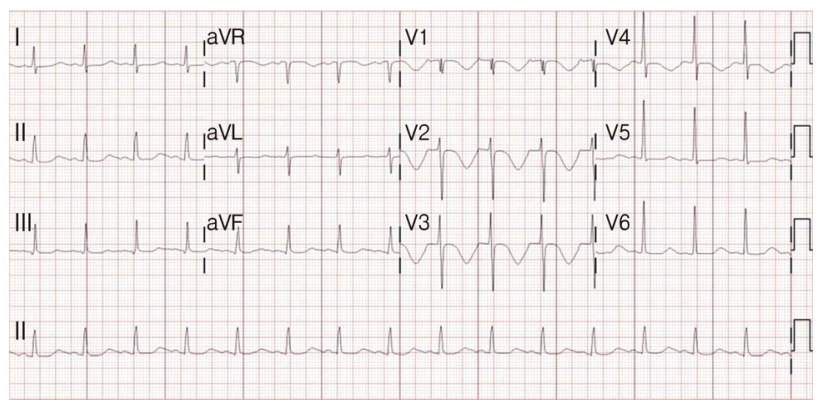

Fig. 1. Twelve-lead electrocardiography taken after the infusion of intravenous amiodarone. Note the negative $\mathrm{T}$ waves in leads V1-V4 and QT prolongation.

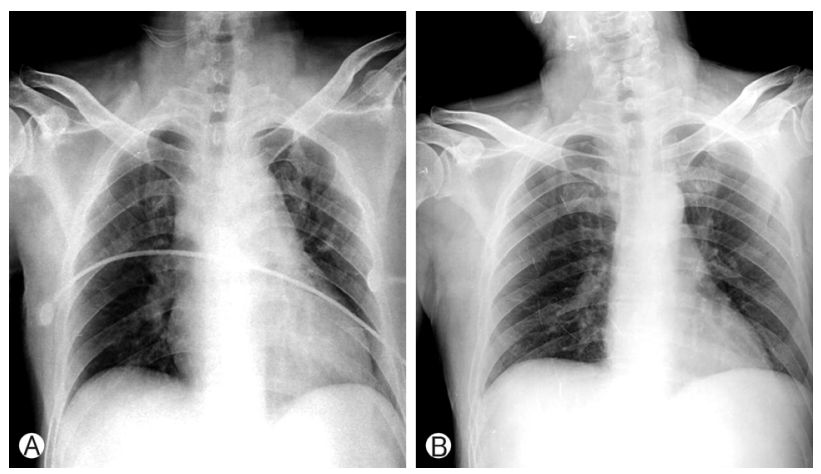

Fig. 2. Chest radiography. (A) Taken at admission, showing marked cardiomegaly. (B) Fourteen days after treatment. Note the improvement of cardiomegaly.

jugular veins were dilated and hepatomegaly was present, but pitting edema was absent in both lower extremities. Central venous pressure was elevated at $25 \mathrm{cmH}_{2} \mathrm{O}$. Initial electrocardiography showed ventricular tachycardia (which was not recorded by electrocardiography in an emergent situation) and after the administration of intravenous amiodarone, this changed to a normal sinus rhythm with negative $\mathrm{T}$ waves in leads V1 to V4 and QT prolongation (Fig. 1). Chest radiography showed a prominent pulmonary trunk and moderate cardiomegaly with cardio-thoracic ratio of $57 \%$ (Fig. 2A). Laboratory tests showed elevated levels of liver enzymes, blood urea nitrogen and creatinine, indicating hepatic and renal failure (Table 1). Marked increase in serum lactic acid (9.7 $\mathrm{mmol} / \mathrm{L})$ and pro-B type natriuretic peptide $(23,251 \mathrm{pg} / \mathrm{mL})$ levels were also noted. Arterial blood gas analysis indicated respiratory alkalosis with an arterial $\mathrm{pH}$ of $7.605, \mathrm{pCO}_{2}$ of $19.3 \mathrm{~mm} \mathrm{Hg}, \mathrm{pO}_{2}$ of $120.3 \mathrm{~mm} \mathrm{Hg}, \mathrm{HCO}_{3}^{-}$of $19.3 \mathrm{mmol} / \mathrm{L}$, and a base excess of $-2.4 \mathrm{mmol} / \mathrm{L}$. Echocardiography showed marked dilatation of the right ventricle. The right ventricular (RV) end-diastolic internal dimension was $45 \mathrm{~mm}$. Flattening
Table 1 . Laboratory values at admission and during hospitalization

\begin{tabular}{llll}
\hline & \multicolumn{1}{c}{ Day 1 } & \multicolumn{1}{c}{ Day 5 } & Day 14 \\
\hline WBC $(\mu \mathrm{L})$ & 9,960 & 6,180 & 4,430 \\
Hemoglobin $(\mathrm{g} / \mathrm{dL})$ & 9.8 & 8.7 & 9.7 \\
Platelet $(\mu \mathrm{L})$ & 256,000 & 251,000 & 182,000 \\
AST/ALT (U/L) & $459 / 96$ & $132 / 97$ & $68 / 45$ \\
TB/DB (mg/dL) & $3.49 / 2.69$ & $1.24 / 0.95$ & $0.27 / 0.23$ \\
Tp/Alb (g/dL) & $6.5 / 3.7$ & $6.6 / 3.3$ & $7.0 / 3.4$ \\
PT (INR) & 1.13 & & \\
BUN/Cr (mg/dL) & $50.4 / 2.83$ & $20.3 / 0.84$ & $11.8 / 0.5$ \\
Na/K (mmol/L) & $120 / 2.5$ & $146 / 4.7$ & $137 / 3.4$ \\
CRP (mg/dL) & 0.68 & & \\
Lactic acid (mmol/L) & 9.7 & & 1.8 \\
proBNP (pg/mL) & 23,251 & 1,251 & 510 \\
\hline
\end{tabular}

Hepatic and renal failure was improved after initiating intravenous thiamine, and serum levels of lactic acid and proBNP was also improved.

WBC, white blood cell; AST, aspartate aminotransferase; ALT, alanine aminotransferase; TB, total bilirubin; DB, direct bilirubin; $\mathrm{Tp}$, total protein; Alb, albumin; PT, prothrombin time; INR, international normalized ratio; BUN, blood urea nitrogen; $\mathrm{Cr}$, creatinine; $\mathrm{Na}$, sodium; $\mathrm{K}$, potassium; CRP, C-reactive protein; proBNP, prohormone-brain natriuretic peptide.

of the interventricular septum with a D-shaped deformation of the left ventricle was evident. The left ventricular (LV) end-diastolic internal dimension was $42 \mathrm{~mm}$ and the LV ejection fraction was 55\%. Calculated cardiac output was elevated at $7.5 \mathrm{~L} / \mathrm{min}$, indicating a hyperdynamic circulation. The $\mathrm{E}$ of E' ratio was 12 indicating somewhat increased LV enddiastolic pressure (LVEDP). Moderate tricuspid regurgitation was found and maximal tricuspid valve regurgitation velocity was up to $3.46 \mathrm{~m} / \mathrm{sec}$. His estimated RV systolic pressure was $52 \mathrm{~mm} \mathrm{Hg}$ (Fig. 3). There was no evidence of pulmonary thromboembolism by contrastenhanced chest computerized tomography (Fig. 4).

Because of his confused mentality and history of chronic alcohol intake, a neurological disorder due to thiamine deficiency was initially suspected and $100 \mathrm{mg}$ of intravenous thiamine was administered followed by daily intravenous thiamine at $100 \mathrm{mg}$.

Twenty-four hours after administering the intravenous thiamine, his mental status improved and his urine output increased, and within a week, his renal and hepatic functions were normalized. A chest radiography taken at 14 days after admission showed improvement of the cardiomegaly (Fig. 2B). 

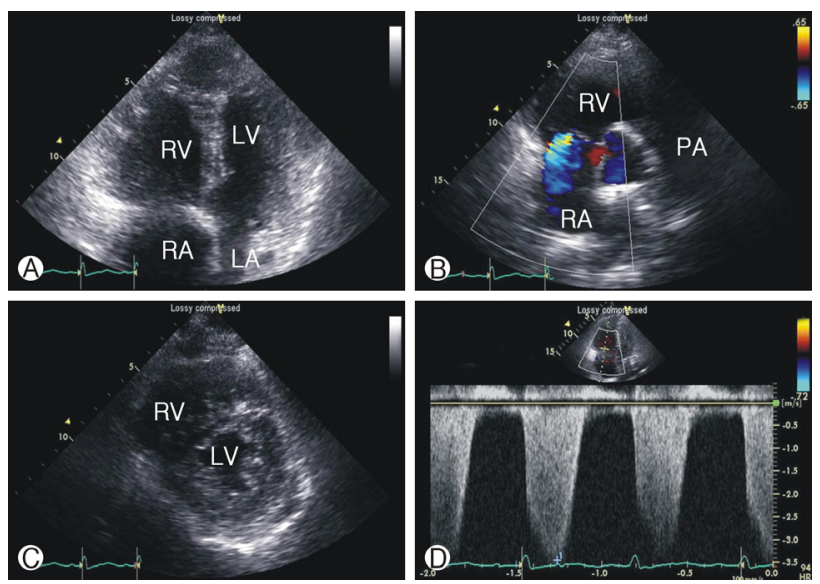

Fig. 3. Two-dimensional echocardiography obtained at admission. (A) Apical 4-chamber view showing marked dilatation of right ventricle. (B) Parasternal short axis view (aortic valve plane) showing moderate tricuspid valve regurgitation. (C) Parasternal short axis view (mid-ventricular plane) showing a flattened interventricular septum with D-shaped deformation of the left ventricle. (D) Maximal velocity of tricuspid valve regurgitation was up to $3.46 \mathrm{~m} / \mathrm{sec}$ and estimated right ventricular systolic pressure was $52 \mathrm{~mm} \mathrm{Hg}$. RV, right ventricle; LV, left ventricle; RA, right atrium; LA, left atrium; PA, pulmonary artery.

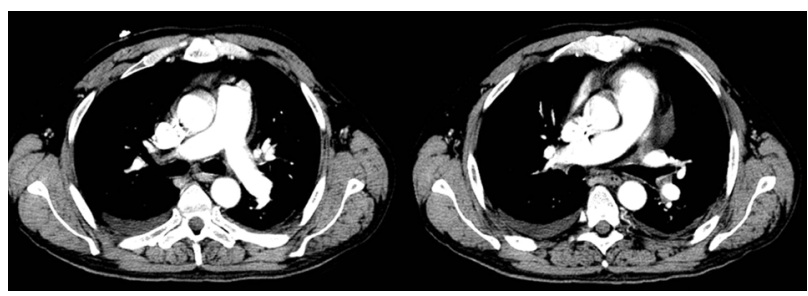

Fig. 4. Contrast-enhanced chest computerized tomography showing no evidence of pulmonary thromboembolism.

Two-dimensional echocardiography at 11 days after admission revealed marked reduction in the size of the right ventricle and markedly improved right ventricular function. The right ventricular end-diastolic internal dimension was decreased at $23 \mathrm{~mm}$. The previously flattened interventricular septum and a D-shaped deformation of the left ventricle were no longer evident. Tricuspid valve regurgitation was reduced and estimated right ventricular systolic pressure had decreased to $13 \mathrm{~mm} \mathrm{Hg}$ (Fig. 5). He was discharged without dyspnea after 3 weeks of treatment. However, his ataxic gait had not fully recovered and he continues to be followed up by our neurologic department.

\section{DISCUSSION}
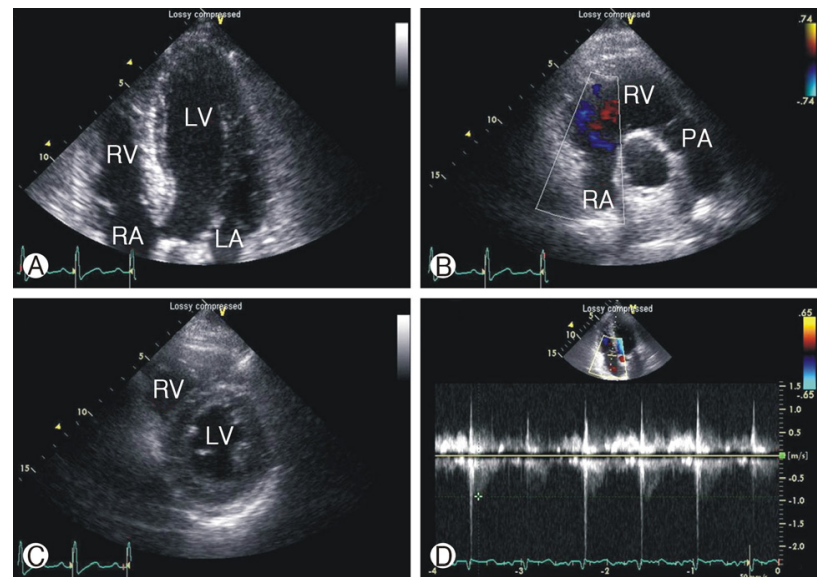

Fig. 5. Two-dimensional echocardiography obtained 11 days after admission. (A) Apical 4-chamber view showing marked reduction of the right ventricle. (B) Parasternal short axis view (aortic valve plane) showing marked improvement of tricuspid valve regurgitation. (C) Parasternal short axis view (mid-ventricular plane) showing improvement of the D-shaped deformation of the left ventricle. (D) The velocity of tricuspid valve regurgitation was markedly reduced and estimated right ventricular systolic pressure had decreased to $13 \mathrm{~mm} \mathrm{Hg}$. LV, left ventricle; RV, right ventricle; $\mathrm{RA}$, right atrium; LA, left atrium; PA, pulmonary artery.

We hereby report a case of pulmonary hypertension with right-sided heart failure, which showed marked improvement after the administration of intravenous thiamine, and was finally diagnosed as cardiovascular beriberi. This condition is one of the causes of reversible form of heart failure, and can occur in those on an inadequate diet or with an excessive alcohol intake [6]. Thiamine is a cofactor of several essential enzymes, such as, $\alpha$-ketoglutarate dehydrogenase, pyruvate dehydrogenase, and transketolase, in the Krebs cycle and the pentose phosphate pathway [7].

Alcohol consumption may result in alcohol related cardiomyopathy by 2 basic mechanisms: a thiamine deficiency (cardiovascular beriberi) and a direct toxic effect of alcohol (alcoholic cardiomyopathy) [8].

Alcoholic cardiomyopathy (ACM) is caused by long-term heavy alcohol consumption and it is the leading cause of non-ischemic, dilated cardiomyopathy [9] ACM occurs in 2 stages: an asymptomatic stage and a symptomatic stage. The asymptomatic stage of ACM is characterized by LV dilatation, increased LV mass, and diastolic dysfunction. Whereas the symptomatic ACM stage shows pronounced LV dilatation, increased LV mass, wall thinning, systolic dysfunction with low cardiac output, and signs and symptoms of heart failure. 
Those who continue to drink may become symptomatic ACM [10]. The pathologic findings of ACM are characterized by hydropic degeneration, interstitial edema, focal necrosis and varying degrees of fibrosis. The smaller branches of the coronary arteries show degenerative and occlusive changes [11]. ACM is distinguishable from cardiovascular beriberi by its lack of response to thiamine. Alcohol abstinence is a cornerstone of this disease and medical therapy is not different from that for other etiologies of heart failure.

In contrast to $\mathrm{ACM}$, cardiovascular beriberi is due to thiamine deficiency and it is curable by correcting this deficiency. It is hemodynamically characterized by high cardiac output failure associated with arteriolar vasodilatation [12]. Increased pulmonary arterial blood flow and elevated LVEDP can be a cause of pulmonary hypertension [13]. Various electrocardiogram abnormalities, including biphasic or inverted T-waves, ST-segment elevation, and QT prolongation have been reported [14]. On the contrary to ACM, the LV function of the beriberi heart has been reported to be normal or hyperkinetic [5]. It is associated with predominantly rightsided heart failure and rapidly recovered after treatment with thiamine. In addition, the myocardium of beriberi heart shows little damage because the disturbance is biochemical rather than structural [11].

The diagnosis of cardiovascular beriberi is difficult because of its rarity, laboratory constraints, and the lack of tests suitable for use in emergency situations. A standard laboratory diagnosis of thiamine deficiency is made by assessing erythrocyte transketolase activity at baseline and after thiamine administration [14,15], but this test is non-specific and is rarely performed in clinical practice. Rather, a therapeutic trial of intravenous thiamine, which improves the adverse hemodynamic situation rapidly, is diagnostic of this rare disease, and thus, our patient was diagnosed based on his response to thiamine. Erythrocyte transketolase activity could not be measured due to limitations of our facilities.

For diagnosis and follow up after treatment of cardiovascular beriberi, echocardiography is most useful imaging modality. It is handy and can be performed in the emergency situation. Also it can evaluate pulmonary hypertension quantitatively and precisely [14]. In this case, initial pulmonary hypertension was found by dilatation of the right ventricle and flattening of the interventricular septum with a D-shaped deformation of the left ventricle. Tricuspid valve regurgitation was also important finding. These findings lead us to rule out the other cause of pulmonary hypertension such as acute pulmonary thromboembolism, so we performed contrast-enhanced chest computerized tomography which showed no evidence of occlusion of pulmonary artery. While continuing therapy with thiamine, we frequently followed up the patient's situation by echocardiography which showed improvement of pulmonary hypertension. As a result, reversible acute pulmonary hypertension was evidently diagnosed and by considering the patient's history of chronic alcoholism we could conclude the cardiovascular beriberi as a final diagnosis.

Recently thiamine deficiency is a rare clinical condition because of the improvement of nutritional status along with marked economic development. Although in 2006, Kwon et al. reported shoshin beriberi, severe form of cardiovascular beriberi characterized as hypotension, tachycardia, lactic acidosis, and anuria, caused by thiamine deficiency [16], national report of this uncommon disease is rare nowadays in Korea according to our literature survey. A high index of suspicion is needed to make a correct diagnosis. Thiamine deficiency must be included in the differential diagnosis of right sided heart failure with pulmonary hypertension, when there is a high risk of nutritional deficiency such as chronic alcoholism, because a thiamine infusion can dramatically restore this rare but important disease. In this case, thiamine deficiency was initially suspected because of a confused mentality and a history of chronic alcohol intake. Thiamine was promptly administered and pulmonary hypertension and right-sided heart failure rapidly improved.

In conclusion, thiamine deficiency can still be encountered in the clinical setting. Thus, prompt suspicion followed by quick administration of thiamine is crucial to reverse pulmonary hypertension in cardiovascular beriberi and echocardiography is important modality for the diagnosis and follow up after treatment.

\section{REFERENCES}

1. Corcoran TB, O'Hare B, Phelan D. Shoshin beri-beri precipitated by intravenous glucose. Crit Care Resusc 2002;4:31-4.

2. Astudillo L, Degano B, Madaule S, Sailler L, Galinier A, Couret B, et al. Development of beriberi heart disease 20 years after gastrojejunostomy. Am J Med 2003;115:157-8.

3. Oliveira FA, Guatimosim S, Castro CH, Galan DT, LautonSantos S, Ribeiro AM, et al. Abolition of reperfusion-induced 
arrhythmias in hearts from thiamine-deficient rats. Am J Physiol Heart Circ Physiol 2007;293:H394-401.

4. Roman-Campos D, Campos AC, Gioda CR, Campos PP, Medeiros MA, Cruz JS. Cardiac structural changes and electrical remodeling in a thiamine-deficiency model in rats. Life Sci 2009;84:817-24.

5. Park JH, Lee JH, Jeong JO, Seong IW, Choi SW. Thiamine deficiency as a rare cause of reversible severe pulmonary hypertension. Int J Cardiol 2007;121:e1-3.

6. Kawano H, Hayashi T, Koide Y, Toda G, Yano K. Histopathological changes of biopsied myocardium in Shoshin beriberi. Int Heart J 2005;46:751-9.

7. Bello S, Neri M, Riezzo I, Othman MS, Turillazzi E, Fineschi V. Cardiac beriberi: morphological findings in two fatal cases. Diagn Pathol 2011;6:8.

8. Pinn G, Bovet P. Alcohol-related cardiomyopathy in the Seychelles. Med J Aust 1991;155:529-32.

9. Piano MR. Alcoholic cardiomyopathy: incidence, clinical characteristics, and pathophysiology. Chest 2002;121:1638-50.

10. George A, Figueredo VM. Alcoholic cardiomyopathy: a review. J Card Fail 2011;17:844-9.

11. Gubbay ER. Beri-Beri heart disease. Can Med Assoc J 1966; 95:21-7.

12. Jeffrey FE, Abelmann WH. Recovery from proved Shoshin beriberi. Am J Med 1971;50:123-8.

13. Okura H, Takatsu Y. High-output heart failure as a cause of pulmonary hypertension. Intern Med 1994;33:363-5.

14. Yamasaki H, Tada H, Kawano S, Aonuma K. Reversible pulmonary hypertension, lactic acidosis, and rapidly evolving multiple organ failure as manifestations of shoshin beriberi. Circ J 2010;74:1983-5.

15. Camilo ME, Morgan MY, Sherlock S. Erythrocyte transketolase activity in alcoholic liver disease. Scand J Gastroenterol 1981;16:273-9.

16. Kwon TJ, Hwang JY, Park SR, Kang YR, Lee HY, Kwak CH, et al. A case of shoshin beriberi presenting as acute coronary syndrome with shock: shoshin beriberi mimicking acute coronary syndrome. J Cardiovasc Ultrasound 2006;14:116-9. Korean. 